\title{
Wntless (Wls): A Prognostic Index for Progression and Patient Survival of Breast Cancer
}

This article was published in the following Dove Press journal: OncoTargets and Therapy

\author{
Dandan Zheng (DiD ${ }^{1,2, *}$ \\ Chengwei Jiang ${ }^{3, *}$ \\ Ning Yan $^{2}$ \\ Yayun Miao ${ }^{2}$ \\ Keren Wang ${ }^{2}$ \\ $\mathrm{Ge} \mathrm{Gao}^{3}$ \\ Yan Jiao iD ${ }^{4}$ \\ Xiangkai Zhang $\mathbb{D}^{2}$ \\ Miao $\mathrm{He}^{5}$ \\ Zhaoying Yang $\mathbb{D}^{2}$
}

'Department of Gastroenterology, Sun Yat-sen Memorial Hospital, Sun Yat-sen University, Guangzhou, Guangdong 5 I0120, People's Republic of China; ${ }^{2}$ Department of Breast Surgery, ChinaJapan Union Hospital of Jilin University, Changchun, Jilin I30033, People's

Republic of China; ${ }^{3}$ Department of Pathology, China-Japan Union Hospital of Jilin University, Changchun, Jilin I30033, People's Republic of China; ${ }^{4}$ Department of Hepatobiliary and Pancreatic Surgery, The First Hospital of Jilin University, Changchun, Jilin 13002I, People's Republic of China; ${ }^{5}$ Department of Anesthesia, The Second Hospital of Jilin University, Changchun, Jilin I30022, People's Republic of China

*These authors contributed equally to this work

Correspondence: Miao He

Department of Anesthesia, The Second Hospital of Jilin University, 218 Ziqiang

Street, Changchun, Jilin I30022, People's Republic of China

Email hemiao@jlu.edu.cn

Zhaoying Yang

Department of Breast Surgery, ChinaJapan Union Hospital of Jilin University,

I 26 Xiantai Street, Changchun, Jilin

130033, People's Republic of China

Email zyyang@jlu.edu.cn
Background: Wntless (Wls) is an essential protein that is necessary for the secretion of Wnt proteins. While numerous researches have demonstrated that aberrations in $\mathrm{Wnt} / \beta$-catenin expression lead to tumorigenesis and progression in many cancer types, the effects of Wls in breast cancer (BC) are less studied.

Methods: The mRNA and protein expression of Wls in BC cell lines were detected by RTqPCR and Western blot; the protein expression of patient samples was detected by immunohistochemistry (IHC). The associations between Wls expression and clinicopathological factors as well as survival time, including overall survival (OS) and disease-free survival (DFS) were analyzed. Bioinformatics analysis was used to reveal the correlation between Wls genes and associated genes or pathways.

Results: Wls was overexpressed in BC cell lines and tissues. The expression level of Wls was significantly correlated with tumor size, estrogen receptor (ER), progesterone receptor (PR), Ki-67, molecular classification, and follow-up status. Spearman correlation analysis showed that Wls protein expression was negatively correlated with ER and PR, which was confirmed by bioinformatics analysis in mRNA level. However, there were positive relationships with MBNG (modified Black's nuclear grade), tumor size, Ki-67, molecular classification, follow-up, and vital status. Univariate and multivariate analysis showed that Wls was an independent prognostic factor for OS and DFS in BC patients. Moreover, Wls was a significant prognostic indicator of OS and DFS in a hormone receptor-positive (HR+) subgroup. GSEA showed that estrogen and androgen response, as well as epithelialmesenchymal transition pathways, were up-regulated in the Wls high-expression group.

Conclusion: Overexpression of Wls is a significant marker of worse prognosis in $\mathrm{BC}$ and might play a crucial role in the HR+ subgroup.

Keywords: wntless, estrogen receptor, progesterone receptor, androgen receptor, breast cancer, prognosis

\section{Introduction}

Breast cancer (BC), is the most common malignant tumor among female patients, and has become the first cause of cancer-related deaths globally. The increasing BC incidence is projected to increase further in the next 20 years worldwide. ${ }^{1}$ More than 400,000 women die of BC every year despite the use of multimodal treatments. ${ }^{2}$ Therefore, it is crucial to identify specific bio-markers for early diagnosis and prognosis of BC patients.

As a complex signaling pathway, the Wnt pathway has been reported to be participated in cell proliferation, differentiation, apoptosis, and polarity. ${ }^{3}$ Numerous studies have revealed that abnormalities of Wnt signaling are closely related to the 
development of human tumors, including BC. ${ }^{4,5}$ Wntless (Wnt Ligand Secretion Mediator, Wls), also known as Sprinter (SRT), Evenness interrupted (EVI), or GPR177, is a regulatory molecule, which plays a crucial role in the secretion of Wnt proteins. ${ }^{6-8} \mathrm{Wls}$ is a transmembrane protein, ${ }^{6}$ found to be indispensable for Wnt proteins exocytosis and transport. ${ }^{9}$ Banziger's study found that Wls played an important role in $\mathrm{Wg}$-sending rather than $\mathrm{Wg}$ receiving cells in Drosophila melanogaster. ${ }^{8}$ Studies in human cells indicated that Wls function was required in cell-to-cell communication mediated by the Wnt pathway. Wls has received increasing attention and its expression and function have been reported in various human cancer types, including malignant astrocytomas, gastric cancer, and ovarian cancer. ${ }^{4,10,11} \mathrm{Lu}$ reported that Wls regulated cell proliferation in $\mathrm{BC}$ by adjusting the Wnt signaling. ${ }^{5}$ However, the prognostic value of Wls in BC is less studied. In this study, the expression of Wls in BC samples was detected using IHC, and the correlation between clinicopathological parameters and Wls expression was analyzed. The present study revealed that Wls was overexpressed in BC cells and tissues. Furthermore, Wls overexpression was associated with poor prognosis in $\mathrm{BC}$ patients, and may be an independent risk factor of $\mathrm{BC}$. Additionally, the hormone receptor-positive (HR+) $\mathrm{BC}$ subgroup, which defined as estrogen receptor (ER) $\geq 10 \%$ and/or progesterone receptor (PR) $\geq 10 \%$ by IHC staining, showed that high level of Wls was related to poor OS and DFS of patients.

\section{Methods}

\section{Breast Cell Lines}

The human BC cells, MDA-MB-453 were grown in Leibovitz's L-15 (Thermo Fisher, MA, USA), MDA-MB -468 were grown in RPMI 1640, MCF-7 and ZR-75 were cultured in DMEM medium (Hyclone, UT, USA), supplemented with $10 \%$ fetal bovine serum (Gibco NY, USA). All human BC cell lines were obtained from the American Type Culture Collection (VA, USA). MDA-MB-453 were cultured without $\mathrm{CO}_{2}$ equilibration while other cell lines were cultured in a humid atmosphere of $5 \% \mathrm{CO}_{2}$ at $37{ }^{\circ} \mathrm{C}$.

\section{Real-Time Quantitative Polymerase Chain Reaction (RT-qPCR)}

Total RNA was isolated by TRIzol (Ambion, CA, USA). Reverse transcription was carried out using the $5 \times$ PrimeScript RT Master Mix for Real-Time (TaKaRa,
Tokyo, Japan) and RT-qPCR was performed by $\mathrm{SYBR}^{\circledR}$ Premix Dimer Eraser ${ }^{\mathrm{TM}}$ Perfect Real-Time (TaKaRa, Tokyo, Japan) following the manufacturer's instructions. The RT-qPCR analysis was performed using Mastercycler (Eppendorf, Hamburg, Germany). The relative gene expression fold change was normalized using GAPDH as an internal control and compared with MCF-10A. The sequences of used primers in this study were as follows: Wls, F: 5'-TCA TGG TAT TTC AGG TGT TTC G-3', R: 5'-GCA TGA GGA ACT TGA ACC TAA AA-3'; GAPDH, F: 5'-GAA GGC TGG GGC TCA TTT GCA GGG-3', R: 5'-GGT GCA GGA GGC ATT GCT GAT GAT- $3^{\prime}$.

\section{Western Blot}

For Western blot, antibodies against Wls, ER $\alpha, \operatorname{ER} \beta$, and GAPDH were purchased from Proteintech (IL, USA), PR and AR (androgen receptor) were purchased from Abcam (Cambridge, UK). Western blot analysis was performed as follows. Briefly, BC cells were lysed with RIPA buffer, and the cell lysates centrifuged $\left(12,000 \times \mathrm{g}, 10 \mathrm{~min}, 4^{\circ} \mathrm{C}\right)$, to collect the supernatant proteins. Protein concentration was determined by the Bio-Rad method (Hercules, CA, USA). A total of $25 \mu \mathrm{g}$ protein were loaded onto the SDSPAGE gels $(10 \%)$ and subsequently transferred to nitrocellulose membranes, blocked with 5\% skim milk at room temperature for 1 hour, and incubated at $4^{\circ} \mathrm{C}$ overnight, with Wls, ER $\alpha$, ER $\beta$, PR, AR or GAPDH anti-rabbit antibodies at a dilution of 1:1000. Each membrane was then incubated with an anti-rabbit horseradish peroxidase antibody (Proteintech, IL, USA) at room temperature for 1h. Detection of immunoreactivity was carried out using enhanced chemiluminescence (Millipore, Germany) and visualization performed using an autoradiography imager G: Box Chemi XT4 System (Syngene, Cambridge, UK).

\section{Patients and Tissue Samples}

A total of $247 \mathrm{BC}$ and 40 para-tumor paraffin-embedded tissues were collected from patients with complete clinicopathological and follow-up information who were diagnosed with $\mathrm{BC}$ and surgically treated at China-Japan Union Hospital of Jilin University between 2012 and 2014. Subsequently, IHC analysis was performed on all tissue samples collected from the Department of Pathology. Before surgery, patients received no radiotherapy or chemotherapy. All cases were histologically diagnosed and scored by 2 independent pathologists based on the WHO criteria. We defined OS as the duration between 
the time of surgery and death or last follow-up and calculated DFS from the date of surgery until initial tumor relapse or last follow-up.

\section{Immunohistochemistry Staining (IHC)}

IHC was performed as previously reported in our experiment. ${ }^{12}$ Briefly, $3 \mu \mathrm{m}$ thick sections were deparaffinized, rehydrated, and submerged in EDTA for antigenic repair. The sections were next dealt with hydrogen, heated, and incubated in bovine serum albumin. The cells were incubated with the Wls antibody (Proteintech, IL, USA) at $4^{\circ} \mathrm{C}$ overnight. Normal goat serum served as a negative control. The sections were washed and subsequently incubated with secondary antibody, and horseradish peroxidase-streptavidin complex (Invitrogen, CA, USA). Each section was stained using 3-amino-9-ethyl carbazole followed by counterstaining with Mayer's hematoxylin before dehydration and mounting. Staining intensity of Wls protein was rated as 0 , absent; 1 , weak; 2, moderate; 3 , strong. The percentage of positive cells was grouped as $0(<5 \%), 1(6-10 \%), 2(10-25 \%), 3(25-50 \%)$, or 4 $(>50 \%)$. The total IHC score was determined by the following formula: Positive IHC score = positive percentage score $\times$ positive intensity score. Wls protein expression was classified as low ( score $\leq 3)$, moderate $(3<$ scores $<6$ ), and high (score $\geq 6$ ) expression groups.

\section{Bioinformatic Analysis Gene Set Enrichment Analyze (GSEA)}

The mRNA profile data were obtained from the TCGA database and included $1104 \mathrm{BC}$ tissues and 114 normal tissues. The mRNA level of Wls was divided into a lowlevel or high-level group based on the median ratio. Briefly, gene sets (h.all.v6.2.symbols.gmt) were obtained from the Molecular Signatures Database and were analyzed using GSEA software 3.0. Normalized enrichment scores (NES) were obtained from 1000 site permutations with $p$-value $<0.05$ and FDR (false discovery rates) $<0.25$ were considered statistically significant.

\section{Correlation of Wls and ESRI, PGR, or AR}

The mRNA profile of the normal tissues was acquired from the Genotype-Tissue Expression (GTEx) Project (https://www.gtexportal.org/). The mRNA expression data of $\mathrm{BC}$ tissues and $\mathrm{BC}$ cell lines $(\mathrm{n}=57)$ were respectively downloaded from the TCGA and CCLE portal (https:// portals.broadinstitute.org/ccle/home). The correlation between Wls and estrogen receptor 1 (ESR1), progesterone receptor (PGR), and androgen receptor (AR) was analyzed using the R program (Version 3.5.2; http://www.R-project.org/bioconductor.org/).

\section{Statistical Analysis}

All statistical data were calculated by SPSS software version 23.0 (IBM, NY, USA). The Chi-square test as well as Spearman's correlation analysis was adopted for analysis of the relationship between Wls expression and clinicopathological parameters. Kaplan-Meier method was used to assess survival, and the difference in survival between the groups was determined through the Log-rank test. Further, survival evaluation was subjected to multivariate Cox-regression analysis, following univariate analysis. Data analysis among groups adopted one-way ANOVA. A p-value $<0.05$ signified statistical significance. Each experiment was done at least three times and measured data expressed as mean \pm standard deviation.

\section{Results Wls Expression in BC Cell Lines and Tissues}

Wls was overexpressed in $\mathrm{BC}$ cell lines (Figure 1) and $\mathrm{BC}$ tissues (Figure 2). The mRNA level of Wls, as shown in Figure 1A, was up-regulated in $\mathrm{BC}$ cell lines, especially in basal-like BC MDA-MB-453 and MDA-MB-468. A similar expression pattern of Wls protein expression was obtained by Western blot (Figure 1B) in BC cells, ER and PR status were also detected. Moreover, there was no expression of Wls in most para-tumor breast tissues detected by IHC staining (Figure 2A), while high expression was observed in most BC tissues, which could be divided into low, moderate, and high expression groups (Figure 2B-D).

\section{Relationship Between Clinicopathological Features and Wls Expression of BC}

The clinicopathological parameters of $247 \mathrm{BC}$ patients are shown in Table 1. To assess whether Wls expression was connected with the clinical progression of $\mathrm{BC}$, the Chisquare test was applied to determine the association between Wls expression and clinicopathological parameters. The results showed that the level of Wls was related to tumor size $(p=0.050)$, ER $(p=0.000), \quad P R$ $(\mathrm{p}=0.000), \mathrm{Ki}-67 \quad(\mathrm{p}=0.019)$, molecular classification $(\mathrm{p}=0.000)$ and follow-up status $(\mathrm{p}=0.002)$.

As shown in Table 2, Wls expression in invasive BC showed a positive correlation with MBNG (modified Black's 
A

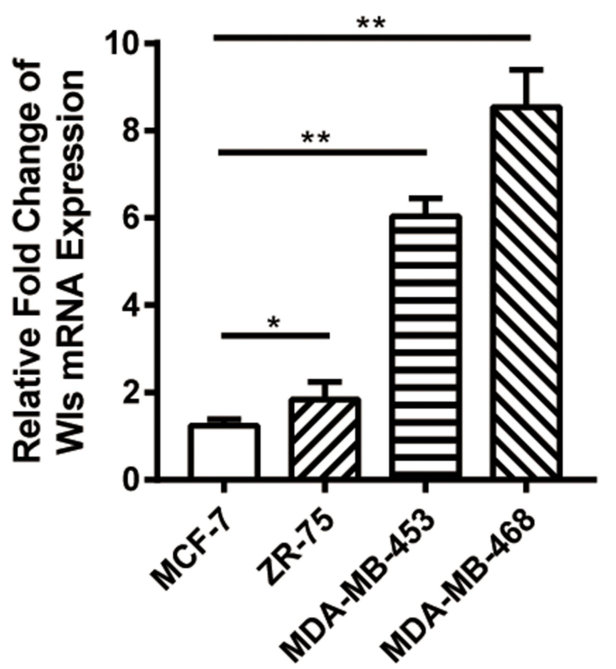

B

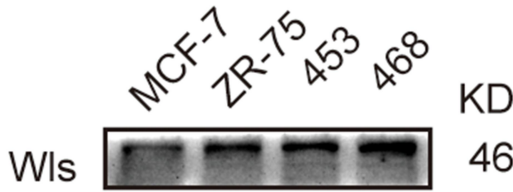

ERa

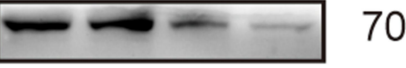

ER $\beta$

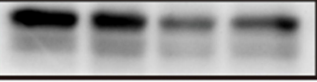

60

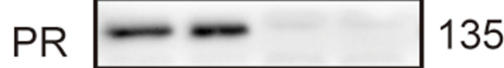

AR

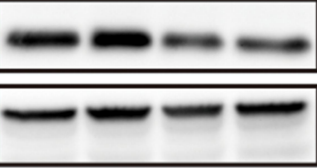

GAPDH

110

36

Figure I Overexpressed Wls in BC cell lines. Wls expression level was detected in both mRNA (A) and protein level (B) in different cell lines. ER $\alpha$, ER $\beta$, PR, and AR were detected by Western blot. $\left(* p<0.05,{ }^{* *} \mathrm{p}<0.01\right)$.

Abbreviations: AR, androgen receptor; BC, breast cancer; ER, estrogen receptor; PR, progesterone receptor; Wls, wntless.

A

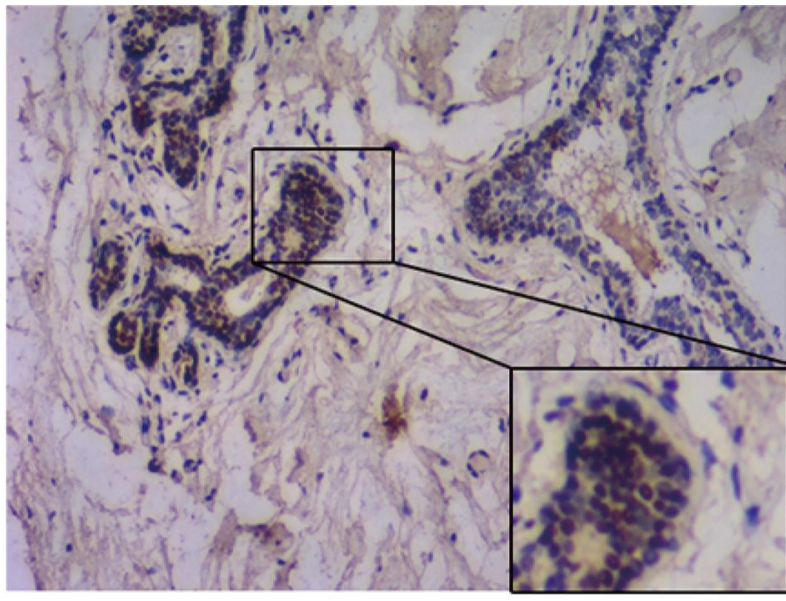

C

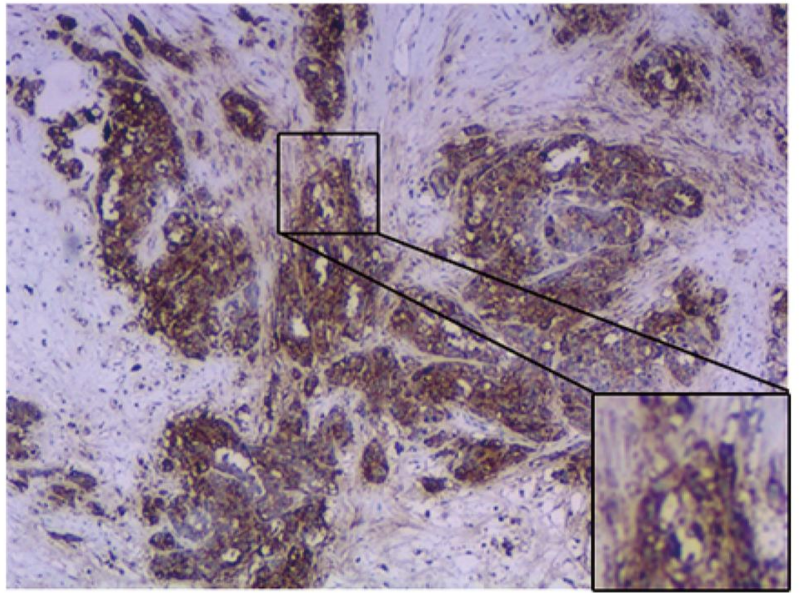

B
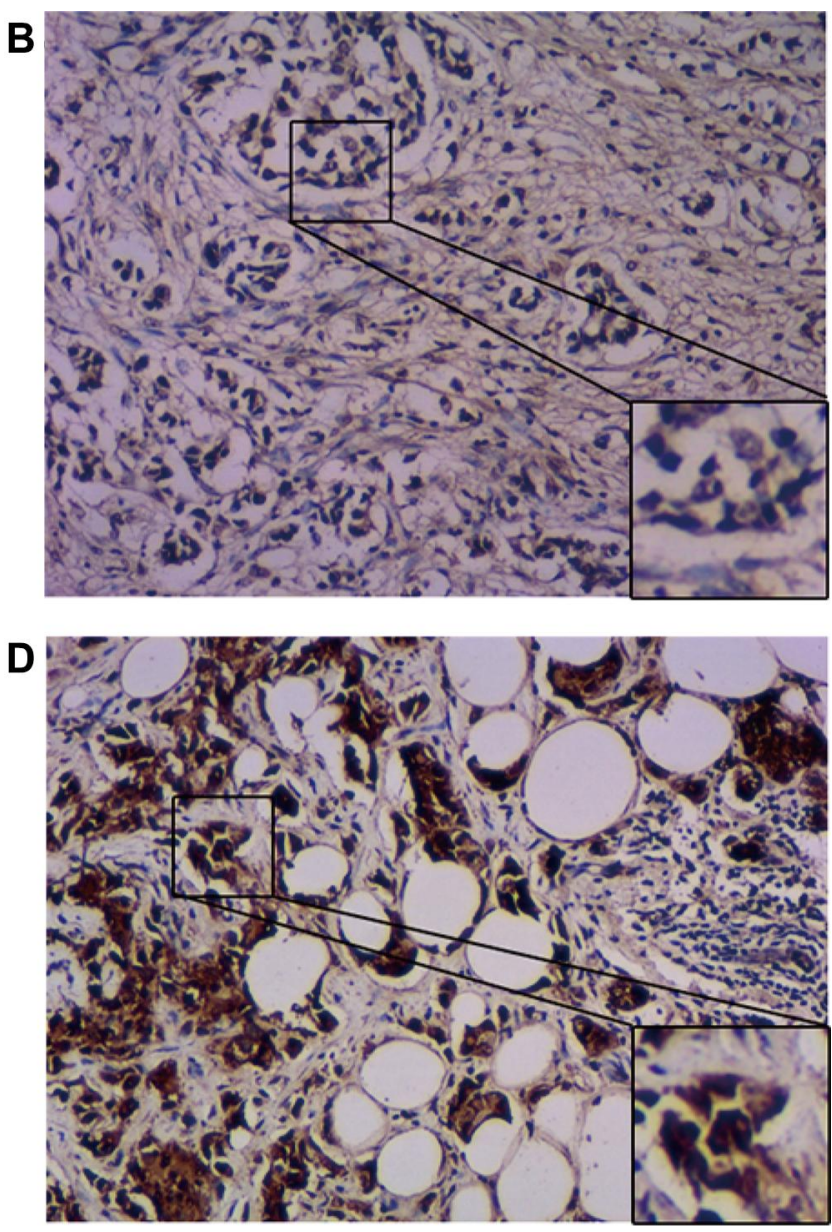

Figure 2 Overexpressed Wls in BC tissues. Immunohistochemistry staining detected the expression of Wls in human tissues. (A) Negative staining; (B) Light staining (I+); (C) Mild staining (2+); (D) Strong staining $(3+)$. The right lower figure is the magnification $(\times 100)$ of the squared area $(\times 40)$.

Abbreviations: BC, breast cancer; Wls, wntless. 
Table I Correlation Between Clinicopathological Characteristics and Wls Expression in Breast Cancer Patient Samples

\begin{tabular}{|c|c|c|c|c|}
\hline \multirow[t]{2}{*}{ Characteristics } & \multicolumn{3}{|c|}{ Wls Expression } & \multirow[t]{2}{*}{ p-value } \\
\hline & Low & Middle & High & \\
\hline \multicolumn{5}{|l|}{ Age (year) } \\
\hline$<50$ & 30 & 68 & 19 & \\
\hline$\geq 50$ & 30 & 79 & 21 & 0.887 \\
\hline \multicolumn{5}{|l|}{ Menopause stage } \\
\hline No & 34 & 80 & 23 & \\
\hline Yes & 26 & 67 & 17 & 0.920 \\
\hline \multicolumn{5}{|l|}{ Age at menarche (year) } \\
\hline$<15$ & 26 & 77 & 20 & \\
\hline$\geq 15$ & 34 & 70 & 20 & 0.498 \\
\hline \multicolumn{5}{|l|}{ Clinical stage } \\
\hline 1 & 21 & 57 & 11 & \\
\hline$\|$ & 25 & 55 & 16 & \\
\hline III & 14 & 35 & 13 & 0.664 \\
\hline \multicolumn{5}{|l|}{ Tumor size(cm) } \\
\hline$<2$ & 40 & 88 & 17 & \\
\hline$\geq 2$ & 20 & 59 & 23 & 0.050 \\
\hline \multicolumn{5}{|l|}{ Number of lymph node metastases } \\
\hline 0 & 30 & 79 & 18 & \\
\hline $1-3$ & 16 & 35 & 10 & \\
\hline 4-9 & 7 & 17 & 4 & \\
\hline$\geq 10$ & 7 & 16 & 8 & 0.828 \\
\hline \multicolumn{5}{|l|}{ ER status } \\
\hline Negative & 5 & 42 & 21 & \\
\hline Positive & 55 & 105 & 19 & 0.000 \\
\hline \multicolumn{5}{|l|}{ PR status } \\
\hline Negative & 9 & 52 & 24 & \\
\hline Positive & 51 & 95 & 16 & 0.000 \\
\hline \multicolumn{5}{|l|}{ Her-2 status } \\
\hline Negative & 33 & 76 & 17 & \\
\hline $1-2+$ & 23 & 60 & 19 & \\
\hline $3+$ & 4 & 11 & 4 & $0.780^{\mathrm{a}}$ \\
\hline \multicolumn{5}{|l|}{ Expression of Ki-67 (30\%) } \\
\hline Low expression & 49 & 95 & 23 & \\
\hline High expression & 11 & 52 & 17 & 0.019 \\
\hline \multicolumn{5}{|l|}{ Molecular classification } \\
\hline Luminal A & 29 & 49 & 4 & \\
\hline Luminal B (Her-2-) & 13 & 34 & 5 & \\
\hline Luminal B (Her-2+) & 12 & 26 & 10 & \\
\hline Her-2+ & 3 & 21 & 6 & \\
\hline Basal like & 3 & 17 & 15 & 0.000 \\
\hline
\end{tabular}

(Continued)
Table I (Continued).

\begin{tabular}{|l|l|l|l|l|}
\hline \multirow{2}{*}{ Characteristics } & \multicolumn{3}{|c|}{ Wls Expression } & \multirow{2}{*}{ p-value } \\
\cline { 2 - 4 } & Low & Middle & High & \\
\hline Follow-up status & & & & \\
Alive & 53 & 118 & 24 & \\
Metastasis & 2 & 8 & 0 & \\
Recurrence & 0 & 1 & 2 & \\
Dead & 5 & 20 & 14 & $0.002^{\mathrm{a}}$ \\
\hline
\end{tabular}

Notes: ${ }^{\text {a}}$ Fisher's exact test.

Abbreviations: $E R$, estrogen receptor; Her-2, human epidermal growth factor-2; $\mathrm{M}$, metastasis; N, node; PR, progesterone receptor.

Table 2 Spearman Analysis of Correlation Between Wls and Clinicopathological Features

\begin{tabular}{|l|l|l|}
\hline \multirow{2}{*}{ Characteristics } & \multicolumn{2}{|l|}{ Wls Expression Level } \\
\cline { 2 - 3 } & Spearman Correlation & $p$-value \\
\hline MBNG & 0.129 & 0.042 \\
Tumor size & 0.150 & 0.018 \\
ER status & -0.308 & 0.000 \\
PR status & -0.294 & 0.000 \\
Expression of Ki67(30\%) & 0.173 & 0.006 \\
Molecular classification & 0.331 & 0.000 \\
Follow-up status & 0.212 & 0.001 \\
Vital status & 0.210 & 0.001 \\
\hline
\end{tabular}

Abbreviations: ER, estrogen receptor; Her-2, human epidermal growth factor-2; M, metastasis; MBNG, modified Black's nuclear grade; N, node; PR, progesterone receptor.

nuclear grade, $\mathrm{r}=0.129, \mathrm{p}=0.042)$, tumor size $(\mathrm{r}=0.150$, $\mathrm{p}=0.018)$, Ki-67 $(\mathrm{r}=0.173, \mathrm{p}=0.006)$, molecular classification $(\mathrm{r}=0.331, \mathrm{p}=0.000)$, follow-up status $(\mathrm{r}=0.212, \mathrm{p}=0.001)$ and vital status $(\mathrm{r}=0.210, \mathrm{p}=0.001)$, while showed a negative correlation with ER $(r=-0.308, p=0.000)$ and $\mathrm{PR}(\mathrm{r}=-0.294$, $\mathrm{p}=0.000$ ).

\section{Wls Expression in BC Patients is Related to Poor Prognosis}

The impact of Wls expression on patients' survival compared with Ki-67 was evaluated by Kaplan-Meier and logrank analysis (Figure 3). The relationship between Wls expression and OS and DFS was statistically significant $(p<0.001, p<0.001$; Figure $3 A$ and B). When patients were classified into subgroups based on hormone receptor, Wls was considered as a significant prognostic indicator for OS and DFS in the HR+ subgroup ( $\mathrm{p}=0.010 ; \mathrm{p}=0.004)$ (Figure 

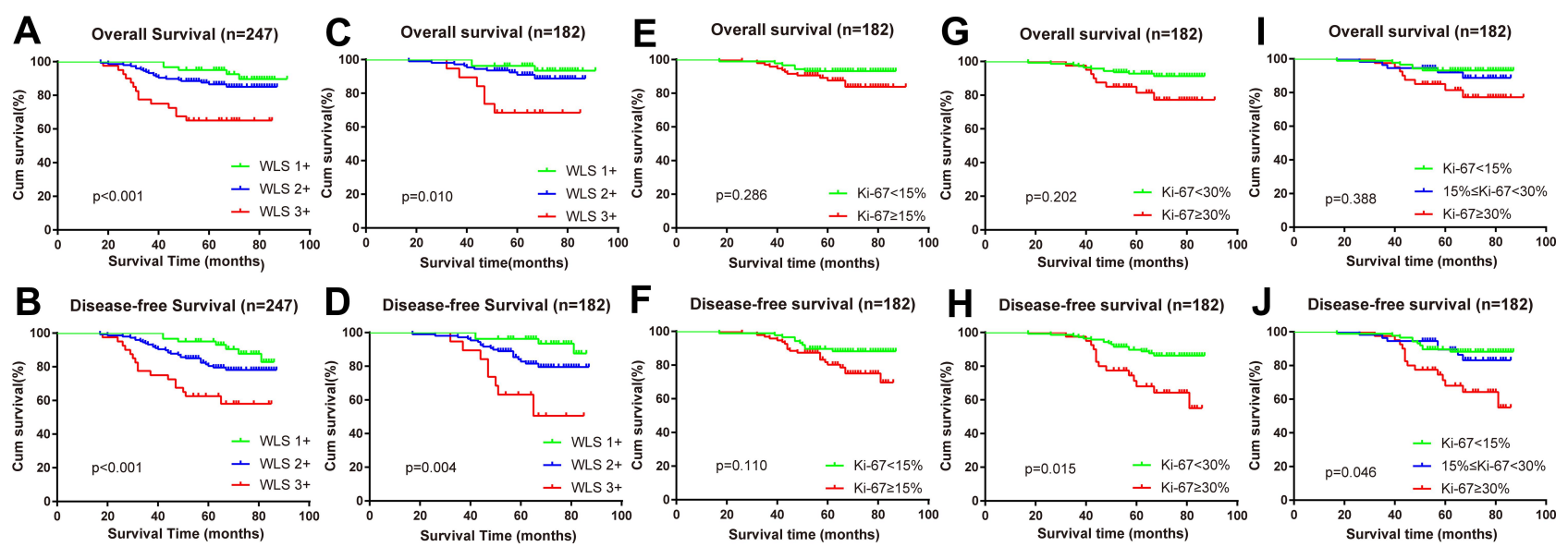

Figure 3 High expression of Wls correlates with OS and DFS and compares with the expression of Ki-67 in different cut-off values. (A, B) OS and DFS of the whole samples. (C, D) OS and DFS of the sub-group of HR+ patients. (E-J) OS and DFS of the sub-group of HR+ patients were divided as the expression of Ki-67 with a cut-off value of $15 \%(\mathbf{E}, \mathbf{F}), 30 \%(\mathbf{G}, \mathbf{H})$, or a combination $(\mathbf{I}, \mathbf{J})$.

Abbreviations: DFS, disease-free survival; $\mathrm{HR+}$, hormone receptor-positive; OS, overall survival; Wls, wntless.

$3 \mathrm{C}$ and D). However, Ki-67 (cut-off value at $15 \%, 30 \%$ or 15-30\%) showed no statistical significance with OS (Figure 3E, G, and I) in the HR+ subgroup and was only related to poor DFS when its expression was $\geq 30 \%$ (Figure 3F, H and J). As shown in Table 3, for patients with high expression of Wls, the median OS and DFS times were 67.125 months (HR: 3.953, 95\% CI: 59.378 to 74.872 ) and 65.357 months (HR: $3.937,95 \%$ CI: 57.640 to 73.075), respectively. For patients with the moderate expression of Wls, the median OS and DFS times were 80.094 months (HR: $1.459,95 \%$ CI: 77.234 to 82.953 ) and 77.720 months (HR: $1.577,95 \%$ CI: 74.629 to 80.812 ), respectively, and the median OS and DFS times of patients with low expression of Wls were 87.501 months (HR: 1.514, $95 \%$ CI: 84.533 to 90.468 ) and 82.340 months (HR: $1.370,95 \%$ CI: 79.655 to 85.024 ), respectively.

\section{Univariate and Multivariate Survival Analysis of BC Patients}

Univariate and multivariate analysis were used to confirm the prognostic value of related clinicopathological parameters. As shown in Table 4, univariate analysis showed that age at menarche $(\mathrm{p}=0.013)$, Wls expression $(p<0.001)$, clinical stage $(p<0.001)$, tumor size $(p<0.001)$, $\mathrm{N}$ stage $(\mathrm{p}<0.001)$, ER $(\mathrm{p}<0.001)$, PR $(\mathrm{p}<0.001), \mathrm{Ki}-67$ $(p=0.008)$, Her-2 $(p=0.024)$ and molecular classification $(p<0.001)$ were all significant prognostic factors for OS. Besides, only Wls expression ( $\mathrm{p}=0.023$ ), as well as $\mathrm{N}$ stage $(\mathrm{p}=0.024)$, were independent prognostic predictors in multivariate analysis, and all the other factors showed no independent prognostic effect. In univariate analysis, Wls expression, age at menarche, clinical stage, tumor size, $\mathrm{N}$ stage, ER, PR, Ki-67, and molecular classification, were significantly related to DFS $(\mathrm{p}<0.001)$ in BC patients (Table 5). Besides, high Wls and Ki-67 expression, low PR expression, and high $\mathrm{N}$ stage were associated with poor DFS in multivariate analysis $(\mathrm{p}=0.002)$.

\section{Relationship Between Wls Expression and Hormone Receptor}

Bioinformatics analysis was employed to explore the correlation between Wls and ESR1, PGR, or AR and the

Table 3 Log-Rank Analysis of Overall Survival (OS) and Disease-Free Survival (DFS) in Different Expression Groups of Wls in Breast Cancer Patients

\begin{tabular}{|l|l|l|l|l|l|l|}
\hline \multirow{2}{*}{ Wls Expression } & \multicolumn{2}{l}{ OS } & \multicolumn{2}{l|}{ DFS } \\
\cline { 2 - 7 } & Months & HR & $\mathbf{9 5 \%} \mathbf{~ I ~}$ & Months & HR & $\mathbf{9 5 \%} \mathbf{~ C I}$ \\
\hline \multirow{2}{*}{+} & 87.501 & 1.514 & $84.533-90.468$ & 82.340 & 1.370 & $79.655-85.024$ \\
$2+$ & 80.094 & 1.459 & $77.234-82.953$ & 77.720 & 1.577 & $74.629-80.812$ \\
$3+$ & 67.125 & 3.953 & $59.378-74.872$ & 65.357 & 3.937 & $57.640-73.075$ \\
Total & 82.370 & 1.288 & $79.846-84.894$ & 77.147 & 1.248 & $74.701-79.592$ \\
\hline
\end{tabular}


Table 4 Univariate and Multivariate Cox-Regression Analysis of Various Prognostic Parameters of Overall Survival (OS) in Patients with Breast Cancer

\begin{tabular}{|c|c|c|c|c|c|c|}
\hline \multirow[t]{2}{*}{ Characteristics } & \multicolumn{3}{|c|}{ Univariate Analysis } & \multicolumn{3}{|c|}{ Multivariate Analysis } \\
\hline & HR & $95 \% \mathrm{Cl}$ & p-value & HR & $95 \% \mathrm{Cl}$ & p-value \\
\hline Age at menarche & 1.235 & $1.046-1.459$ & 0.013 & 1.175 & $0.974-1.417$ & 0.092 \\
\hline Wls & 2.560 & $1.537-4.263$ & 0.000 & 1.972 & $|.099-3.54|$ & 0.023 \\
\hline Clinical stage & 2.593 & $1.656-4.058$ & 0.000 & 0.788 & $0.273-2.28 I$ & $0.66 \mathrm{I}$ \\
\hline Tumor size & 2.454 & $1.577-3.817$ & 0.000 & 1.469 & $0.834-2.588$ & 0.183 \\
\hline $\mathrm{N}$ stage & 1.989 & $1.522-2.599$ & 0.000 & 2.000 & $1.096-3.650$ & 0.024 \\
\hline ER status & 0.309 & $0.165-0.580$ & 0.000 & 1.269 & $0.376-4.290$ & 0.701 \\
\hline PR status & 0.221 & $0.114-0.431$ & 0.000 & 0.353 & $0.124-1.003$ & 0.051 \\
\hline Ki-67 status ( $30 \%)$ & 2.339 & I.248-4.383 & 0.008 & 1.314 & $0.638-2.706$ & 0.458 \\
\hline Her-2 status & 1.694 & $1.072-2.679$ & 0.024 & 1.107 & $0.712-1.720$ & 0.652 \\
\hline Molecular classification & 1.483 & I.197-1.838 & 0.000 & 1.048 & $0.646-1.702$ & 0.848 \\
\hline
\end{tabular}

Abbreviations: $\mathrm{Cl}$, confidence interval; ER, estrogen receptor; Her-2, human epidermal growth factor-2; HR, hazard ratio; M, metastasis; N, node; PR, progesterone.

Table 5 Univariate and Multivariate Cox-Regression Analysis of Various Prognostic Parameters of Disease-Free Survival (DFS) in Patients with Breast Cancer

\begin{tabular}{|c|c|c|c|c|c|c|}
\hline \multirow[t]{2}{*}{ Characteristics } & \multicolumn{3}{|c|}{ Univariate Analysis } & \multicolumn{3}{|c|}{ Multivariate Analysis } \\
\hline & HR & $95 \% \mathrm{Cl}$ & p-value & HR & $95 \% \mathrm{Cl}$ & p-value \\
\hline Age at menarche & 1.163 & $1.004-1.347$ & 0.044 & 1.120 & $0.949-1.322$ & 0.181 \\
\hline Wls & 2.267 & $1.467-3.505$ & 0.000 & 2.149 & $1.313-3.518$ & 0.002 \\
\hline Clinical stage & 2.213 & $1.520-3.223$ & 0.000 & 0.591 & $0.24 I-1.450$ & 0.251 \\
\hline Tumor size & 1.962 & $1.325-2.904$ & 0.001 & 1.193 & $0.729-1.950$ & 0.483 \\
\hline $\mathrm{N}$ stage & 1.900 & $1.506-2.395$ & 0.000 & 2.532 & $1.469-4.362$ & 0.001 \\
\hline ER status & 0.479 & $0.275-0.834$ & 0.009 & 2.264 & $0.778-6.583$ & 0.134 \\
\hline PR status & 0.344 & $0.199-0.596$ & 0.000 & 0.407 & $0.176-0.944$ & 0.036 \\
\hline Ki-67 status ( $30 \%)$ & 2.486 & $1.442-4.285$ & 0.001 & 1.949 & $1.030-3.688$ & 0.040 \\
\hline Her-2 status & 1.397 & $0.930-2.098$ & 0.107 & & & \\
\hline Molecular classification & 1.337 & $1.114-1.603$ & 0.002 & 1.083 & $0.726-1.615$ & 0.696 \\
\hline
\end{tabular}

Abbreviations: $\mathrm{Cl}$, confidence interval; ER, estrogen receptor; Her-2, human epidermal growth factor-2; HR, hazard ratio; M, metastasis; N, node; PR, progesterone receptor.

effects of Wls on tumor progression. GSEA showed that estrogen response (Figure 4A), epithelial-mesenchymal transition (EMT) (Figure 4B), and androgen response (Figure 4C) pathways were up-regulated in the Wls highexpression group. The correlation between Wls mRNA expression and ESR1 or PGR showed an inverse relationship among normal tissues (GTEx, $\mathrm{r}=0.59, \mathrm{p}<0.01 ; \mathrm{r}=0.71$, $\mathrm{p}<0.01$, Figure $4 \mathrm{D}$ and $\mathrm{G}$ ) and $\mathrm{BC}$ tissues (TCGA, $\mathrm{r}=0.18$, $\mathrm{p}<0.05 ; \mathrm{r}=0.37, \mathrm{p}<0.01$, Figure $4 \mathrm{E}$ and $\mathrm{H})$ and $\mathrm{BC}$ cell lines (CCLE, $r=-0.41, \mathrm{p}<0.001 ; \mathrm{r}=-0.27, \mathrm{p}<0.05$, Figure 4F and I). Besides, the correlation between Wls and AR (Figure $4 \mathrm{~J}-\mathrm{L}$ ) showed an inverse relationship between $\mathrm{BC}$ tissues $(\mathrm{r}=0.34, \mathrm{p}<0.001$, Figure $4 \mathrm{~K})$ and $\mathrm{BC}$ cell lines $(\mathrm{r}=$ $-0.25, \mathrm{p}=0.06$, Figure $4 \mathrm{~L}$ ) but the relationship was not statistically significant.

\section{Discussion}

In this study, we found that Wls was overexpressed in BC cells and tissues. Wls overexpression was also found to be an independent risk factor of poor prognosis in $\mathrm{BC}$ patients, including the HR+ subgroup.

Numerous studies have demonstrated aberrant expression of Wls in multi-types of tumors. Zhou found that Wls was highly expressed in hepatocellular carcinoma and showed positive correlation with tumor differentiation and TNM stage. ${ }^{13}$ A study by Augustin, showed that Wls was required for glioma tumorigenesis and progression, which was related to poor prognosis in various cancer types. ${ }^{11} \mathrm{Xu}$ found that overexpression of Wls was an independent prognostic indicator, and was closely related to poor outcomes of colorectal cancer patients. ${ }^{14}$ 

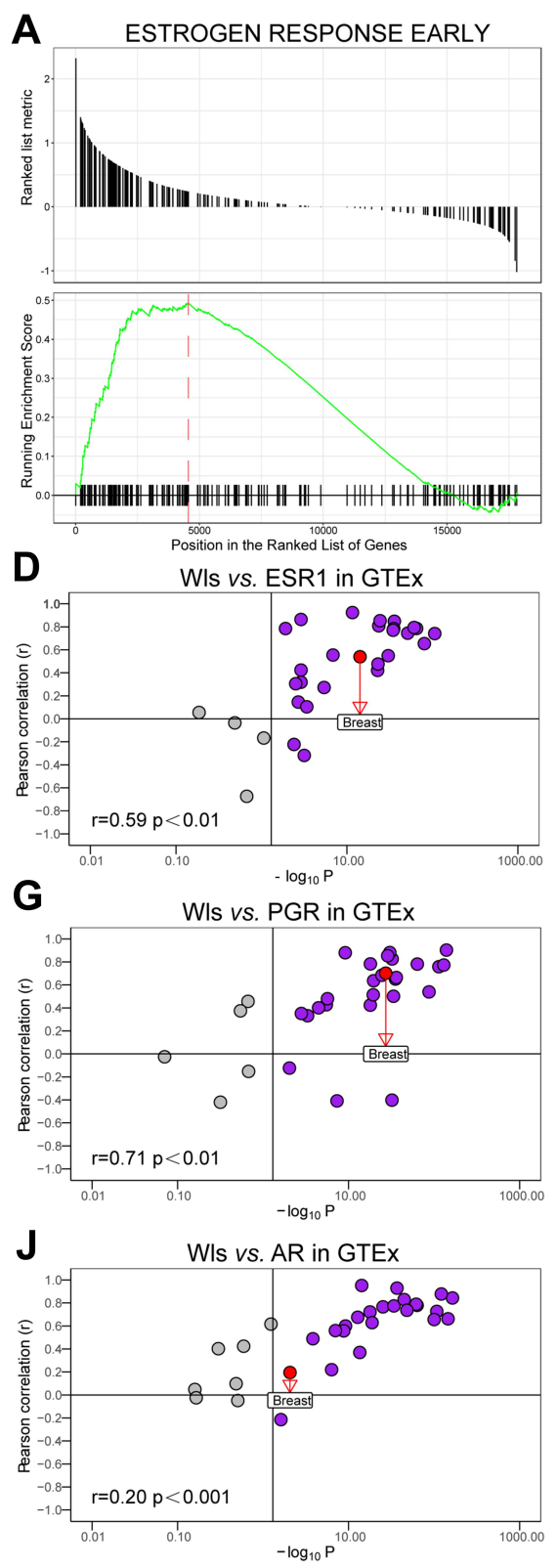
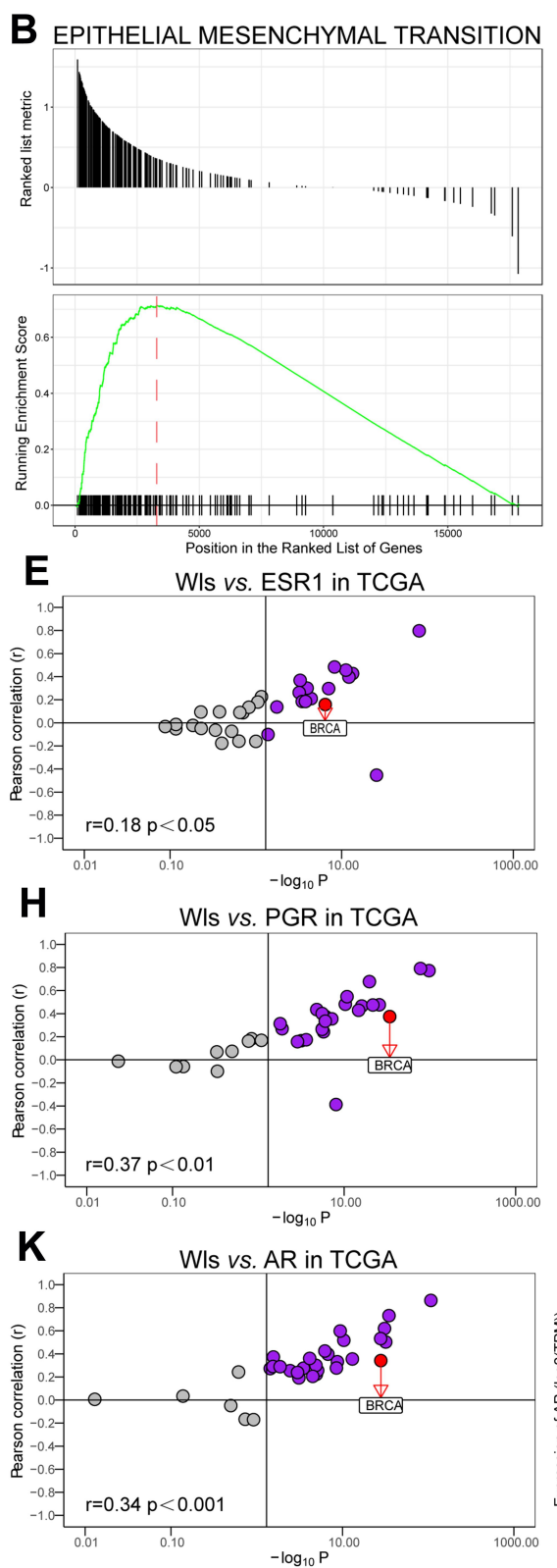
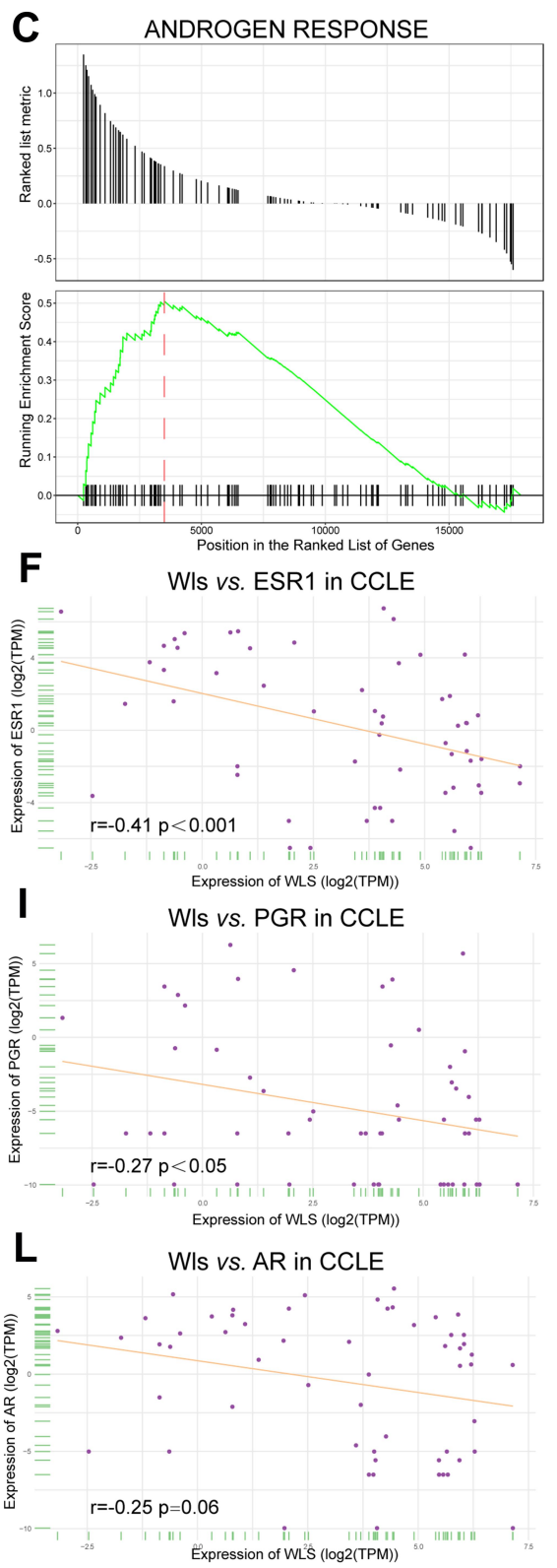

Figure 4 Bioinformatic analysis of the associated pathways and genes. GSEA show that estrogen response (A), epithelial mesenchymal transition (B), and androgen response (C) pathways are upregulated in the Wls high-expression group. The correlation between Wls and ESRI, PGR or AR is analyzed in the GTEx (D, G, and J), TCGA (E, H, and K), and CCLE $(\mathbf{F}, \mathbf{I}$, and $\mathbf{L})$ databases.

Abbreviations: AR, androgen receptor; EMT, epithelial-mesenchymal transition; ESRI, estrogen receptor I; GSEA, gene set enrichment analysis; PGR, progesterone receptor; Wls, wntless.

Emerging evidence suggests that the secretion of Wnts is controlled by Wls, which combined with the FZD and LRP5/6 receptor complex on the cell surface, stimulates cell adhesion, proliferation, differentiation, apoptosis, EMT and invasion by inducing the canonical (Wnt/ $\beta$-catenin) or non-canonical (Wnt $/ \mathrm{Ca}^{2+}$ ) signaling pathway. ${ }^{15-18}$ Previous studies demonstrated that the Wnt pathway was closely associated with breast carcinogenesis. ${ }^{19-21}$ However, only a few studies reported a relationship between Wls expression status and breast carcinoma. ${ }^{5,22}$
This study found that elevated Wls protein expression was negatively correlated with ER and PR status. These findings were consistent with the results in a study by Stewart, that Wls was overexpressed in breast, gastric and ovarian cancers. Wls expression is significantly positively correlated with HER-2 and negatively correlated with ER and PR. ${ }^{22}$ In our present study, the mRNA expression of ESR1 and PGR showed a strong positive correlation with Wls expression in normal tissues, a relatively weak positive correlation in $\mathrm{BC}$ tissues, and a negative correlation in $\mathrm{BC}$ 
cell lines. These findings indicate that Wls links closely to the progression of $\mathrm{BC}$, and high expression of Wls is associated with poor prognosis in $\mathrm{BC}$ patients.

Moreover, $\mathrm{HR}+\mathrm{BC}$ was considered to have a better prognosis than the Her-2 positive and basal-like subtype. Wls was detectable in the HR+ cell lines MCF-7 and ZR75. The expression of Wls was found to increase in the more invasive and aggressive basal-like $\mathrm{BC}$ cell lines MDA-MB-453 and 468. We also found that overexpression of Wls in the $\mathrm{HR}+$ subtype correlated with poor prognosis. Song et al found that activation of $\mathrm{Wnt} / \beta$ catenin signaling in luminal cells promotes basal transition under pregnancy or estrogen and progesterone treatment, which may explain the basal-like cancer cells with high expression of Wls. ${ }^{23}$ Chen et al reported that $\beta$-catenin was significantly up-regulated, while ER $\alpha$ was down-regulated in hepatocellular carcinoma (HCC). The binding of ER $\alpha$ with $\beta$-catenin was enhanced by estrogen, which activated the binding of $\beta$-catenin with E3 ligase to promote its degradation and block the metastasis in HCC cells. ${ }^{24}$ Considering that liver cancer is more prevalent in males, the possible mechanism associated with a negative correlation between Wls and ER-negative breast cancer still needs to be further investigated.

Furthermore, GSEA showed that estrogen response and EMT pathways were upregulated in the Wls highexpression group. These results were in line with the experimental results reported by other researchers. Kouzmenko et al showed the cross-link between Wnt and estrogen pathways through the interaction between $\mathrm{ER} \alpha$ and $\beta$-catenin even in the absence of ligand in vivo, which was markedly stimulated by estrogen at physiological concentrations. ${ }^{25}$ Sikora et al reported that Wnt4 knockdown blocked estrogen-induced proliferation in invasive lobular carcinoma, which may mediate resistance to endocrine therapy. ${ }^{26}$ Bleckmann et al reported that, in $\mathrm{BC}$ patients with liver metastasis, Wnt signaling linked with poor prognosis of patients. ${ }^{27}$ Leung et al found that the expression of components of Wnt pathway was increased in MCF-7 cell after short period of endocrine treatment. $^{28}$ Mastroianni et al reported that Wnt signaling can function as estrogen to drive mammary epithelial cells growth including $\mathrm{ER} \alpha+$ cells, which suggested that Wnt signaling may contribute to estrogen-independent tumor growth, and resistance of endocrine therapy. ${ }^{29}$ These results suggested a cross-talk between these two important pathways in promoting tumor progression. Interestingly, Mullin identified that Wls and ESR1 were associated with bone density by bioinformatic study. ${ }^{30}$ Besides, the correlation between Wls and ER may involve in various diseases, and the interaction between Wls and ER needs to be further investigated.

Published data reveals that $\mathrm{AR}$ is a novel marker in breast carcinogenesis. AR is reportedly associated with early-stage, low axillary burden, ER, PR, and luminal A molecular subtype. ${ }^{31}$ GSEA displayed that androgen response is enriched in the Wls high-expression group. The correlation between mRNA expression of Wls and $\mathrm{AR}$ showed an inverse relationship between the normal and $\mathrm{BC}$ cells, while no significant relationship was observed between the $\mathrm{BC}$ tissue and $\mathrm{BC}$ cell lines. Since the interaction between $\beta$-catenin and AR has been previously observed, ${ }^{32}$ it is necessary to explore the interaction mechanism between Wls and AR in future studies.

Five to 10 years of adjuvant endocrine therapy is advised in $\mathrm{HR}+$ postmenopausal BC women. ${ }^{33}$ However, while it provides further protection, it also induces the extra risk of side effects associated with therapy beyond 5 years. ${ }^{34}$ In this study, Wls was found to be a significant prognostic indicator of OS and DFS in the HR+ subgroup. Therefore, this is a potentially new predictive biomarker for $\mathrm{HR}+$ patients carrying a high risk of recurrence. These findings also illustrate the benefits of extended endocrine therapy to improve the prognosis in patients without recurrence 5 years after diagnosis and whether treatment can be sustained if an adverse event occurs. ${ }^{34,35}$

The luminal A and B subtypes were characterized by the expression level of $\mathrm{Ki}-67$ at a cut-off value of $14 \%{ }^{36}$ In fact, the reported cut-offs for Ki-67 ranges from 3.5 to $34 \%$. There is no solid evidence that $\mathrm{Ki}-67$ could work as a valuable biomarker to distinguish which patients may benefit from specific chemotherapy or endocrine therapies. Moreover, Ki-67 is not recommended through guidelines from the American Society of Clinical Oncology as a routine biological marker. ${ }^{37}$ The lack of standardization made it not routine for the determination of Ki-67. There are numerous ongoing studies to find other indicators as alternatives of $\mathrm{Ki}-67$ in subtype determination of BC. ${ }^{38}$ In this study, the expression level of Wls was positively correlated with $\mathrm{Ki}-67$, and Wls was found to be an effective prognostic indicator of OS and DFS in BC or $\mathrm{HR}+$ subgroup patients. Wls can serve as a potential predictor for the survival of $\mathrm{BC}$ including the $\mathrm{HR}+$ subgroup.

In summary, overexpression of Wls is a prognostic predictor for survival in $\mathrm{BC}$ patients and shows an even greater advantage in the $\mathrm{HR}+\mathrm{BC}$ subgroup. 


\section{Abbreviations}

$\mathrm{AR}$, androgen receptor; $\mathrm{BC}$, breast cancer; DFS, diseasefree survival; EMT, epithelial-mesenchymal transition; ESR1, estrogen receptor 1 ; $\mathrm{HR}+$, hormone receptorpositive; GSEA, gene set enrichment analyze; IHC, immunohistochemistry; MBNG, modified Black's nuclear grade; OS, overall survival; PGR, progesterone receptor; Wls, wntless.

\section{Ethics}

This study was approved by the China-Japan Union Hospital ethics committee of Jilin University (No. 2019022604). Each patient gave consent to the use of their data in scientific research and all the patients signed informed consent.

\section{Disclosure}

Dandan Zheng and Chengwei Jiang are co-first authors for this work. The authors report no conflicts of interest in this work.

\section{References}

1. Zhou C, Yang X, Sun Y, Yu H, Zhang Y, Jin Y. Comprehensive profiling reveals mechanisms of SOX2-mediated cell fate specification in human ESCs and NPCs. Cell Res. 2016;26(2):171-189.

2. Ferlay J, Steliarova-Foucher E, Lortet-Tieulent J, et al. Cancer incidence and mortality patterns in Europe: estimates for 40 countries in 2012. Eur J Cancer. 2013;49(6):1374-1403. doi:10.1016/j.ejca.2012. 12.027

3. Ring A, Kim Y-M, Kahn M. Wnt/catenin signaling in adult stem cell physiology and disease.. Stem Cell Rev Rep. 2014;10(4):512-525.

4. Stewart J, James J, McCluggage GW, et al. Analysis of wntless (WLS) expression in gastric, ovarian, and breast cancers reveals a strong association with HER2 overexpression. Mod Pathol. 2015;28(3):428-436.

5. Lu D, Li Y, Liu Q-R, et al. Wls promotes the proliferation of breast cancer cells via Wnt signaling. Med Oncol. 2015;32(5). doi:10.1007/ s12032-015-0585-z

6. Hausmann G, Banziger C, Basler K. Helping wingless take flight: how WNT proteins are secreted. Nat Rev Mol Cell Biol. 2007;8 (4):331-336. doi:10.1038/nrm2141

7. Port F, Basler K. Wnt trafficking: new insights into Wnt maturation, secretion and spreading. Traffic. 2010;11(10):1265-1271. doi:10.11 11/j.1600-0854.2010.01076.x

8. Banziger C, Soldini D, Schutt C, Zipperlen P, Hausmann G, Basler K. Wntless, a conserved membrane protein dedicated to the secretion of Wnt proteins from signaling cells. Cell. 2006;125(3):509-522. doi:10.1016/j.cell.2006.02.049

9. Port F, Kuster M, Herr P, et al. Wingless secretion promotes and requires retromer-dependent cycling of wntless. Nat Cell Biol. 2008;10(2):178-185. doi:10.1038/ncb1687

10. Zhang W, Tao H, Chen X, Sugimura H, Wang J, Zhou P. High expression of Wls is associated with lymph node metastasis and advanced TNM stage in gastric carcinomas. Pathol Int. 2017;67 (3):141-146. doi:10.1111/pin. 12508
11. Augustin I, Goidts V, Bongers A, et al. The Wnt secretion protein Evi/Gpr177 promotes glioma tumourigenesis. EMBO Mol Med. 2012;4(1):38-51. doi:10.1002/emmm.201100186

12. Sun L, He M, Xu N, et al. Regulation of RAB22A by mir-193b inhibits breast cancer growth and metastasis mediated by exosomes. Int J Oncol. 2018;53(6):2705-2714.

13. Zhou C, Sun Y, Guo S, Chen X, Bao G, Wang J. Wls expression correlates with tumor differentiation and TNM stage in hepatocellular carcinoma. Dig Dis Sci. 2018;63(1):166-172. doi:10.1007/s10620017-4823-4

14. Xu H, Jiang W, Zhu F, Zhu C, Wei J, Wang J. Expression of wntless in colorectal carcinomas is associated with invasion, metastasis, and poor survival. Apmis. 2016;124(6):522-528. doi:10.1111/apm.12534

15. Dangfeng Z, Zhaohui G, Xin M, et al. Genetic association study identified a $20 \mathrm{~kb}$ regulatory element in WLS associated with osteoporosis and bone mineral density in Han Chinese. Sci Rep. 2017;7(1):1-6.

16. Nusse R, Clevers H. Wnt/beta-catenin signaling, disease, and emerging therapeutic modalities. Cell. 2017;169(6):985-999. doi:10.1016/ j.cell.2017.05.016

17. Nusse R. Wnts and hedgehogs: lipid-modified proteins and similarities in signaling mechanisms at the cell surface. Development. 2003;130(22):5297-5305. doi:10.1242/dev.00821

18. Lin X, Perrimon N. Dally cooperates with drosophila frizzled 2 to transduce Wingless signalling. Nature. 1999;400(6741):281-284. doi: $10.1038 / 22343$

19. Yan Y, Liu F, Han L, et al. HIF-2 $\alpha$ promotes conversion to a stem cell phenotype and induces chemoresistance in breast cancer cells by activating Wnt and notch pathways. J Exp Clin Cancer Res. 2018;37(1). doi:10.1186/s13046-018-0925-x

20. Yang F, Xiao Z, Zhang S. Knockdown of miR-194-5p inhibits cell proliferation, migration and invasion in breast cancer by regulating the Wnt/ $\beta$-catenin signaling pathway. Int $J$ Mol Med. 2018;42 (6):3355-3363.

21. Lopez Almeida L, Sebbagh M, Bertucci F, et al. The SCRIB paralog LANO/LRRC1 regulates breast cancer stem cell fate through WNT/ $\beta$-catenin signaling. Stem Cell Rep. 2018;11(5):1040-1050. doi:10.1016/j.stemcr.2018.09.008

22. Stewart J, James J, McCluggage GW, et al. Analysis of wntless (WLS) expression in gastric, ovarian, and breast cancers reveals a strong association with HER2 overexpression. Mod Pathol. 2015;28(3):428-436. doi:10.1038/modpathol.2014.114

23. Song W, Wang R, Jiang W, et al. Hormones induce the formation of luminal-derived basal cells in the mammary gland. Cell Res. 2019;29 (3):206-220. doi:10.1038/s41422-018-0137-0

24. Chen YF, Velmurugan BK, Wang HL, et al. Estrogen and ERalpha enhanced beta-catenin degradation and suppressed its downstream target genes to block the metastatic function of HA22T hepatocellular carcinoma cells via modulating GSK-3beta and beta-TrCP expression. Environ Toxicol. 2017;32(2):519-529.

25. Kouzmenko AP, Takeyama K, Ito S, et al. Wnt/beta-catenin and estrogen signaling converge in vivo. J Biol Chem. 2004;279 (39):40255-40258. doi:10.1074/jbc.C400331200

26. Sikora MJ, Jacobsen BM, Levine K, et al. WNT4 mediates estrogen receptor signaling and endocrine resistance in invasive lobular carcinoma cell lines. Breast Cancer Res. 2016;18(1):92. doi:10.1186/ s13058-016-0748-7

27. Bleckmann A, Conradi LC, Menck K, et al. beta-catenin-independent WNT signaling and Ki67 in contrast to the estrogen receptor status are prognostic and associated with poor prognosis in breast cancer liver metastases. Clin Exp Metastasis. 2016;33(4):309-323. doi:10.1007/s10585-016-9780-3

28. Leung EY, Askarian-Amiri ME, Sarkar D, et al. Endocrine therapy of estrogen receptor-positive breast cancer cells: early differential effects on stem cell markers. Front Oncol. 2017;7:184. doi:10.3389/ fonc. 2017.00184 
29. Mastroianni M, Kim S, Kim YC, Esch A, Wagner C, Alexander CM Wnt signaling can substitute for estrogen to induce division of ERalpha-positive cells in a mouse mammary tumor model. Cancer Lett. 2010;289(1):23-31. doi:10.1016/j.canlet.2009.07.012

30. Mullin BH, Walsh JP, Zheng HF, et al. Genome-wide association study using family-based cohorts identifies the WLS and CCDC170/ ESR1 loci as associated with bone mineral density. BMC Genomics. 2016;17:136. doi:10.1186/s12864-016-2481-0

31. Anand A, Singh KR, Kumar S, Husain N, Kushwaha JK, Sonkar AA. Androgen receptor expression in an Indian breast cancer cohort with relation to molecular subtypes and response to neoadjuvant chemotherapy - a Prospective Clinical Study. Breast Care (Basel). 2017;12(3):160-164. doi:10.1159/000458433

32. Ni M, Chen Y, Lim E, et al. Targeting androgen receptor in estrogen receptor-negative breast cancer. Cancer Cell. 2011;20(1):119-131

33. Stearns V, Chapman JA, Ma CX, et al. Treatment-associated musculoskeletal and vasomotor symptoms and relapse-free survival in the NCIC CTG MA.27 adjuvant breast cancer aromatase inhibitor trial. J Clin Oncol. 2015;33(3):265.
34. Pan H, Gray R, Braybrooke J, et al. 20-year risks of breast-cancer recurrence after stopping endocrine therapy at 5 years. $N$ Engl $\mathrm{J} \mathrm{Med}$. 2017;377(19):1836-1846. doi:10.1056/NEJMoa1701830

35. Rueda OM, Sammut SJ, Seoane JA, et al. Dynamics of breast-cancer relapse reveal late-recurring ER-positive genomic subgroups. Nature. 2019;567(7748):399-404. doi:10.1038/s41586-019-1007-8

36. Colozza M, Sidoni A, Piccart-Gebhart M. Value of Ki67 in breast cancer: the debate is still open. Lancet Oncol. 2010;11(5):414-415. doi:10.1016/S1470-2045(10)70089-9

37. Cossetti RJ, Tyldesley SK, Speers CH, Zheng Y, Gelmon KA. Comparison of breast cancer recurrence and outcome patterns between patients treated from 1986 to 1992 and from 2004 to 2008 . J Clin Oncol. 2015;33(1):65-73. doi:10.1200/JCO.2014.57.2461

38. Oddó D, Pulgar D, Elgueta N, et al. Can histological grade and mitotic index replace Ki67 to determine luminal breast cancer subtypes? Asian Pac J Cancer Prev. 2018;19(1):179-183.

\section{Publish your work in this journal}

OncoTargets and Therapy is an international, peer-reviewed, open access journal focusing on the pathological basis of all cancers, potential targets for therapy and treatment protocols employed to improve the management of cancer patients. The journal also focuses on the impact of management programs and new therapeutic agents and protocols on patient perspectives such as quality of life, adherence and satisfaction. The manuscript management system is completely online and includes a very quick and fair peer-review system, which is all easy to use. Visit http://www.dovepress.com/ testimonials.php to read real quotes from published authors. 